\title{
Mortes súbitas em bovinos causadas por Palicourea aeneofusca (Rubiaceae) e Mascagnia rigida (Malpighiaceae) na Zona da Mata Paraibana ${ }^{1}$
}

\author{
Jackson S. de Vasconcelos ${ }^{2}$, Franklin Riet-Correa ${ }^{3 *}$, Antonio Flávio M. \\ Dantas $^{3}$, Rosane M.T. de Medeiros ${ }^{3}$ e Álvaro J. de A. Dantas ${ }^{4}$
}

\begin{abstract}
Vasconcelos J.S., Riet-Correa F., Dantas A.F., Medeiros R.M.T. \& Dantas A.J.A. 2008. [Sudden deaths caused by Palicourea aeneofusca (Rubiaceae) and Mascagnia rigida (Malpighiaceae) in cattle in the Zona da Mata of Paraíba.] Mortes súbitas em bovinos causadas por Palicourea aeneofusca (Rubiaceae) e Mascagnia rigida (Malpighiaceae) na Zona da Mata Paraibana. Pesquisa Veterinária Brasileira 28(10):457460. Hospital Veterinário, CSTR, Universidade Federal de Campina Grande, Patos, PB 58700-000, Brazil. E-mail: franklin.riet@pq.cnpq.br.

This paper reports an outbreak of poisoning by Palicourea aeneofusca in cattle, in the municipality of Jacaraú, and another by Mascagnia rigida in the municipality of Sapé. Both outbreaks occurred in the Zona da Mata of Paraíba, where there are no previous reports of poisoning by toxic plants causing sudden death associated to exercise. The toxicity of both plants was tested in rabbits. The lethal dose was of $3 \mathrm{~g}$ per $\mathrm{kg}$ body weight $(\mathrm{g} / \mathrm{kg})$ for fresh $P$. aeneofusca and $10 \mathrm{~g} / \mathrm{kg}$ for fresh Mascagnia rigida. On the farm where the poisoning by $P$. aeneofusca was observed, M. rigida also was found. The farmer reported that this plant also caused sudden death previously. M. rigida collected on that farm and given dried to rabbits caused death at the dose of $10 \mathrm{gkg}$. The poisoning by $P$. aeneofusca in cattle had been previously reported in the Zona da Mata and Agreste of Pernambuco and east of Bahia. There are also numerous farmers' reports about the occurrence of the poisoning in the Zona da Mata of Alagoas, suggesting that it occurs in the whole coastal region, from Bahia to Paraíba. M. rigida is the most important toxic plant for cattle in the Brazilian semiarid. The results of this paper demonstrate that this plant poisoning also occurs in the tropical wet climate of the Zona da Mata.
\end{abstract}

INDEX TERMS: Toxic plants, Palicourea aeneofusca, Mascagnia rigida, cattle, rabbits, plant poisoning, fluoroacetic acid, cardiac failure.

RESUMO.- Neste trabalho descrevem-se, em bovinos, um surto de intoxicação por Palicourea aeneofusca no município de Jacaraú e um surto de intoxicação por Mascagnia rigida, no município de Sapé. Ambos os surtos ocorreram

\footnotetext{
${ }^{1}$ Recebido em 28 de março, de 2008.

Aceito em 16 de abril de 2008.

Este trabalho faz parte da disssertação do primeiro autor no Curso de Mestrado em Medicina Veterinária de Ruminantes e Eqüídeos, Universidade Federal de Campina Grande (UFCG), Paraíba.

2 Pós-Graduação em Medicina Veterinária de Ruminantes e Eqüídeos, Hospital Veterinário, CSTR, UFCG, Patos, PB 58700-000, Brasil. Email: veterinariojsv@yahoo.com.br

${ }^{3}$ Centro de Saúde e Tecnologia Rural (CSTR), UFCG, Campus de Patos, 58700-000 Patos, PB, Brasil. *Autor para correspondência: franklin.riet@pq.cnpq.br

${ }^{4}$ Médico Veterinário da Agronordeste Produtos Agropecuários Ltda, Rodrigues de Carvalho 154, João Pessoa, PB 58700-000.
}

na zona da Mata Paraibana, onde não havia, anteriormente, informação sobre intoxicação por plantas que causam mortes súbitas associadas ao exercício. A toxicidade de ambas as plantas frescas recém coletadas foi testada em coelhos, sendo a dose letal de $3 \mathrm{~g}$ por $\mathrm{kg}$ de peso animal $(\mathrm{g} / \mathrm{kg})$ para $P$. aeneofusca e de $10 \mathrm{~g} / \mathrm{kg}$ para $M$. rigida. $\mathrm{Na}$ fazenda onde ocorreu o surto de intoxicação por $P$. aeneofusca foi encontrada, também $M$. rigida e o produtor informava que esta tinha causado, anteriormente, mortes de bovinos. $M$. rigida coletada nesta última fazenda foi tóxica na dose de $10 \mathrm{~g} / \mathrm{kg}$ da planta seca. Anteriormente a intoxicação por $P$. aeneofusca tinha sido diagnosticada na Zona da Mata e Agreste Pernambucano e leste da Bahia. Há também numerosos históricos da ocorrência desta intoxicação na Zona da Mata Alagoana, o que sugere sua ocorrência no litoral do nordeste, desde a 
Bahia até Paraíba. M. rigida é a planta tóxica mais conhecida e mais importante do nordeste ocorrendo no sertão e no agreste. Este trabalho comprova que, pelo menos na Paraíba, a intoxicação ocorre, também, na Zona da Mata.

TERMOS DE INDEXAÇÃO: Plantas tóxicas, Palicourea aeneofusca, Mascagnia rigida, bovinos, coelhos, intoxicação por planta, ácido fluoroacético, insuficiência cardíaca.

\section{INTRODUÇÃO}

Palicourea aeneofusca (Rubiaceae) causa mortes súbitas associadas ao exercício no Agreste e Zona da Mata pernambucana, leste da Bahia (Tokarnia et al. 2000) e Alagoas (Riet-Correa 2007, dados não publicados). É comumente conhecida pelos nomes de erva-de-rato (Tokarnia et al. 2000) e papaconha (Riet-Correa 2007, dados não publicados). Experimentalmente é tóxica para bovinos, coelhos (Tokarnia et al. 1983) e caprinos (Passos 1983).

Em condições naturais os bovinos se intoxicam por causa da boa palatabilidade da planta, sem necessariamente ter fome, só basta ter acesso às matas e capoeiras (Tokarnia et al. 1983). Os sinais clínicos em bovinos caracterizam-se por tontura, tremores musculares e queda, seguida de morte. Os coelhos apresentam sonolência e apatia (Tokarnia et al. 1983). Na necropsia não se observam alterações. Na microscopia observa-se, em bovinos, degeneração hidrópico-vacuolar dos túbulos uriníferos e vacuolização discreta dos hepatócitos no fígado. A dose tóxica para bovinos é de $0,75 \mathrm{~g} / \mathrm{kg}$ ( (Tokarnia et al. 1983).

A toxicidade de $P$. aeneofusca foi comprovada experimentalmente para caprinos. A dose letal de folhas frescas de $P$. aeneofusca nesta espécie foi de $0,6 \mathrm{~g} / \mathrm{kg}$. Os sintomas caracterizaram-se por taquicardia, taquipnéia e quedas. A morte ocorreu entre 12 e 15 horas após a administração. Macroscopicamente não foi observada nenhuma lesão digna de nota. Na histologia foi observado apenas degeneração de hepatócitos (Passos 1983).

Mascagnia rigida (Juss.) Griseb. é conhecida popularmente pelos nomes tinguí, timbó e pela-bucho entre outros. É a planta tóxica mais importante do semi-árido, responsável por causar mortes súbitas de bovinos na região Nordeste, nordeste de Minas Gerais e o norte do Espírito Santo. (Tokarnia et al. 2000). Experimentalmente em bovinos a intoxicação por $M$. rigida causou morte com a dose de $12,5 \mathrm{~g} / \mathrm{kg}$ de folhas maduras e com os brotos em floração a dose foi de $21,2 \mathrm{~g} / \mathrm{kg}$. Os sinais clínicos caracterizavam-se por taquicardia, respiração irregular, decúbito esternal evoluindo para decúbito lateral, movimentos de pedalagem, opstótono, tremores musculares, murgidos e morte que variou de 3 min a $2 \mathrm{~h}$ após o início dos sintomas. Na necropsia não foi observada nenhuma lesão significativa. Na histologia foi observada degeneração hidrópico-vacuolar das células do epitélio dos túbulos contornados distais, vacuolização dos hepatócitos na zona mediozonal e vacuolização das fibras cardíacas (Tokarnia et al. 1961, 1994).

M. rigida é tóxica experimentalmente para caprinos
(Paraguassu 1983) e coelhos (Tokarnia et al. 1987). Em coelhos a dose letal foi de $4 \mathrm{~g} / \mathrm{kg}$ das folhas frescas e 0,5$0,125 \mathrm{~g} / \mathrm{kg}$ dos frutos. Os sinais clínicos caracterizaramse por incoordenação, queda, decúbito lateral, alguns emitiam gemidos, a respiração ficava ofegante e logo em seguida morte. Os achados de necropsia mais característicos foram evidenciação de padrão lobular hepático, congestão e congestão pulmonar. Na histologia foram observadas apenas lesões degenerativas e vasculares (Tokarnia et al. 1987).

$\mathrm{Na}$ Paraíba surtos de intoxicação por $M$. rigida tem sido observados em todo estado, exceto na Zona da Mata (Medeiros et al. 2002, Riet-Correa et al. 2006).

O objetivo deste trabalho é descrever surtos de mortes súbitas associadas ao exercício causadas por Palicourea aeneofusca e Mascagnia rigida em bovinos na zona da Mata Paraibana, região na qual não eram conhecidas causas de morte súbita.

\section{MATERIAL E MÉTODOS}

Os dados epidemiológicos e clínicos dos surtos foram obtidos junto aos proprietários em visitas as propriedades onde ocorreram os surtos.

A reprodução experimental em coelhos foi realizada no Hospital Veterinário (HV) do Centro de Saúde e de Tecnologia Rural (CSTR), Campus de Patos, da Universidade Federal de Campina Grande (UFCG). Folhas frescas de Palicourea aeneofusca, coletadas em uma fazenda no Município de Jacaraú, na Zona da Mata Paraibana, onde ocorreram surtos de morte súbita em bovinos, foram administradas a 3 coelhos na dose de $3 \mathrm{~g} / \mathrm{kg}$ de peso animal (Quadro 1). Folhas frescas de $M$. rigida coletadas na mesma fazenda foram administradas a 2 coelhos na dose de $10 \mathrm{~g} / \mathrm{kg}$. Outros 2 coelhos receberam folhas secas e trituradas da mesma planta nas doses de $10 \mathrm{~g} / \mathrm{kg} \mathrm{e}$ outros 2 nas doses de 5g/kg (Quadro 2).

Quadro 1. Intoxicação experimental por $\boldsymbol{P}$. aeneofusca em coelhos, com planta coletada no município de Jacaraú, PB

\begin{tabular}{cccccc}
\hline \multirow{2}{*}{$\begin{array}{c}\text { Coelho } \\
\text { no. }\end{array}$} & $\begin{array}{c}\text { Peso } \\
(\mathrm{kg})\end{array}$ & $\begin{array}{c}\text { Dose } \\
(\mathrm{g} / \mathrm{kg})\end{array}$ & \multicolumn{2}{c}{ Sinais clínicos } & \multirow{2}{*}{ Evoluçãão } \\
\hline 1 & 1,70 & 3 & 02h00min & Duração & \\
\hline 2 & 1,33 & 3 & 00h10min & Morreu \\
3 & 1,27 & 3 & 00h40min & 00h40min & Recuperou-se \\
\hline
\end{tabular}

a Início dos sinais clínicos após administração da planta.

Quadro 2. Intoxicação experimental por Mascagnia rigida em coelhos, com planta coletada no município de Jacaraú, PB

\begin{tabular}{|c|c|c|c|c|c|c|}
\hline \multirow[t]{2}{*}{ Planta } & \multirow{2}{*}{$\begin{array}{c}\text { Coelho } \\
\text { no. }\end{array}$} & \multirow{2}{*}{$\begin{array}{c}\text { Peso } \\
\text { (kg) }\end{array}$} & \multirow{2}{*}{$\begin{array}{l}\text { Dose } \\
\text { (g/kg) }\end{array}$} & \multicolumn{2}{|c|}{ Sinais clínicos } & \multirow[t]{2}{*}{ Evolução } \\
\hline & & & & Início ${ }^{a}$ & Duração & \\
\hline Fresca & 4 & 2 & 10 & $S s^{b}$ & - & - \\
\hline Fresca & 5 & 2 & 10 & Ss & - & - \\
\hline Seca & 6 & 1 & 5 & Ss & - & - \\
\hline Seca & 7 & 1,3 & 5 & Ss & - & - \\
\hline Seca & 8 & 1,2 & 10 & $14 \mathrm{h00min}$ & 00h58min & Morreu \\
\hline Seca & 9 & 1 & 10 & $14 \mathrm{~h} 00 \mathrm{~min}$ & 00h59min & Morreu \\
\hline
\end{tabular}

a Início dos sinais clínicos após administração da planta, b Ss $=$ sem sinais. 
Quadro 3. Intoxicação experimental por Mascagnia rigida em coelhos, com planta coletada no município de Sapé, PB

\begin{tabular}{|c|c|c|c|c|c|c|}
\hline \multirow[t]{2}{*}{ Planta } & \multirow{2}{*}{$\begin{array}{c}\text { Coelho } \\
\text { no. }\end{array}$} & \multirow{2}{*}{$\begin{array}{c}\text { Peso } \\
(\mathrm{kg})\end{array}$} & \multirow{2}{*}{$\begin{array}{l}\text { Dose } \\
\text { (g/kg) }\end{array}$} & \multicolumn{2}{|c|}{ Sinais clínicos } & \multirow[t]{2}{*}{ Evolução } \\
\hline & & & & Início ${ }^{a}$ & Duração & \\
\hline $\mathrm{Fr}$ & 10 & 1,1 & 10 & $11 \mathrm{~h} 30 \mathrm{~min}$ & 10h00min & Morreu \\
\hline Fres & 11 & 1,4 & 10 & $\mathrm{Ss}^{\mathrm{b}}$ & - & - \\
\hline Seca & 12 & 2,1 & 2,5 & Ss & - & - \\
\hline Seca & 13 & 2,3 & 2,5 & Ss & - & - \\
\hline
\end{tabular}

a Início dos sinais clínicos após administração da planta; ${ }^{b}$ Ss = sem sinais.

Folhas frescas de Mascagnia rigida coletadas no município de Sapé, na fazenda onde ocorreu outro surto de mortes súbitas, também na zona da Mata Paraibana, foram administradas a 2 coelhos (10 e 11) na dose de $10 \mathrm{~g} / \mathrm{kg}$ e folhas secas foram administradas a outros 2 coelhos (12 e 13) na dose de 2,5g/kg (Quadro 3). O Coelho 14 serviu como testemunha.

Os coelhos eram pesados e calculados as doses. As folhas frescas de $P$. aeneofusca e $M$. rigida foram colocadas nos cochos das gaiolas e os coelhos as ingeriram espontaneamente.

Para os experimentos com a planta seca, $M$. rigida foi secada ao sol e moída em moinho elétrico. As folhas trituradas foram diluídas em água e administradas por sonda oro gástrica.

Os coelhos que morreram foram necropsiados. Os órgãos das cavidades torácica e abdominal e o sistema nervoso central foram fixados em formalina neutra a $10 \%$, incluídos em parafina, cortados em secções de 4-6um e corados por hematoxilina e eosina.

\section{RESULTADOS}

Um surto de intoxicação por Palicourea aeneofusca ocorreu no mês de agosto de 2007 em uma propriedade no município de Jacaraú, localizado na Zona da Mata Paraibana na microrregião do Litoral Norte. Em um rebanho de 80 bovinos que foram mobilizados, 4 apresentaram cambaleio, tremores musculares e quedas, morrendo logo após. O proprietário relatou que todos os anos ocorriam mortes desse tipo em bovinos e suspeitava de 2 plantas; uma conhecida na região como erva-de-rato e outra como cipó-de-cururú. Em visita realizada à propriedade, em 3 de outubro de 2007, foi encontrada uma Rubiacea de flor amarela e forte odor a salicilato quando amassada, que posteriormente foi identificada com $P$. aeneofusca (Fig.1).

Em outra área da fazenda, distante $10 \mathrm{~km}$ da área onde ocorreram as mortes dos bovinos intoxicados por $P$. aeneofusca, o proprietário informou que existia uma planta conhecida por tinguí que também tinha causado mortes de bovinos em anos anteriores, principalmente no início das chuvas. A planta foi identificada como Mascagnia rigida.

No mês de junho de 2007, em uma fazenda no município de Sapé, na Zona da Mata Paraibana, foram observadas mortes súbitas de 5 bovinos em um rebanho de 50 . Os animais foram colocados em uma área que não tinha sido pastejada com bovinos nesse ano e, após uma semana de permanência nessa área, começaram a morrer. Segundo o gerente da fazenda os bovinos, quando vi-

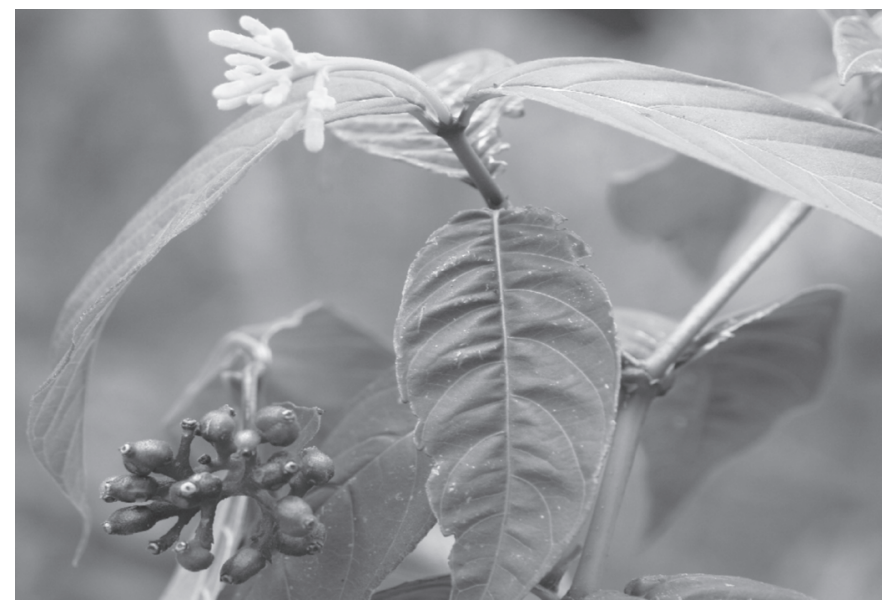

Fig.1. Palicourea aeneofusca (flores, frutos e folhas). Município de Jacaraú, Zona da Mata Paraibana.

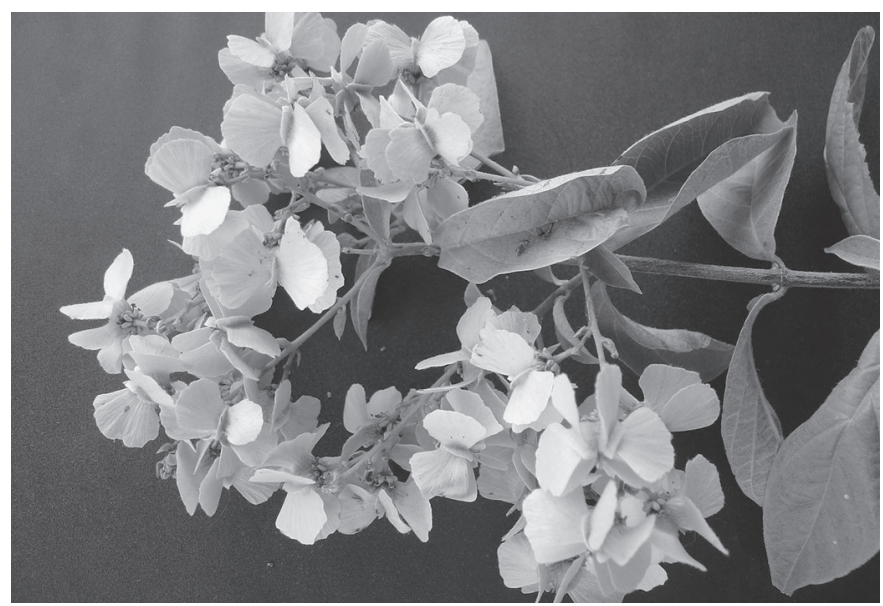

Fig.2. Mascagnia rigida (folhas e frutos). Município de Sapé, Zona da Mata Paraibana.

nham beber água, apresentavam cansaço, tremores e quedas, debatendo-se e morrendo logo em seguida. Em visita a fazenda foi constatada a presença de Mascagnia rigida (Fig.2) na área onde os animais que morreram estavam pastando.

Os resultados da intoxicação experimental em coelhos por $P$. aeneofusca e M. rigida estão descritos nos Quadros 1,2 e 3.

Os Coelhos 1 e 3 apresentaram relutância em caminhar, apatia, aumento da freqüência cardíaca e respiratória, convulsões e gemidos seguidos de morte em 40 e 8 minutos, respectivamente, após o início dos sinais. $\mathrm{Na}$ macroscopia foi observada uma discreta acentuação do padrão lobular hepático. O Coelho 2 apresentou apenas um leve aumento da freqüência cardíaca e respiratória. Os achados histológicos, no Coelho 1, caracterizaram-se por discreta vacuolização de hepatócitos principalmente na região mediozonal. Nos Coelhos 1 e 3, foi observada apenas congestão nas regiões córtico medular e medular do rim, e discreta congestão no coração e pulmão.

Os Coelhos 4, 5, 6, 7, 8 e 9 não apresentaram nenhum 
sinal clínico. Os Coelhos 8 e 9 apresentaram relutância em caminhar, apatia e sonolência, posteriormente se debatiam e apresentavam gemidos, morrendo 59 e 58 minutos, respectivamente, após o início sinais clínicos. Na macroscopia foi observada apenas leve acentuação do padrão lobular hepático. Os achados histológicos foram edema e congestão pulmonar, discreta vacuolização de hepatócitos principalmente na região mediozonal e necrose individual de hepatócitos. No rim foi observada vacuolização do citoplasma das células do epitélio dos túbulos contorcidos distais que também apresentavam núcleo picnótico.

Os sinais clínicos do Coelho 10 foram caracterizados por sonolência, relutância em se exercitar e respiração ofegante; em seguida começou a se debater na gaiola e morreu, tendo uma evolução de 35 minutos após os o início dos sinais. Na necropsia foi observada apenas leve evidenciação do padrão lobular. Não foram observadas lesões significantes na histologia.

\section{DISCUSSÃO}

Os resultados deste trabalho comprovam que tanto a intoxicação por Palicourea aeneofusca, quanto por Mascagnia rigida causam morte súbita em bovinos associada ao exercício na Zona da Mata da Paraíba. Anteriormente, a intoxicação por $P$. aeneofusca tinha sido diagnosticada na Zona da Mata e Agreste Pernambucano e leste da Bahia (Tokarnia et al. 1983, 2000). Há também numerosos históricos da ocorrência desta intoxicação na Zona da Mata Alagoana (Riet-Correa 2007, Universidade Federal de Campina Grande, dados não publicados) o que sugere sua ocorrência em uma extensa área do litoral do nordeste, desde a Bahia até Paraíba.

$M$. rigida é a planta tóxica mais conhecida e mais importante do nordeste ocorrendo no sertão e no agreste (Tokarnia et al. 2000). Este trabalho comprova que, pelo menos na Paraíba, a intoxicação ocorre, também, na Zona da Mata. Um aspecto importante na epidemiologia da intoxicação por $M$. rigida é a marcada variação na sua toxicidade (Tokarnia et al. 2000). Em um trabalho anterior foi comprovado que plantas do agreste foram mais tóxicas que plantas do sertão (Medeiros et al. 2002). Neste traba-
Iho pareceria, também, que a planta coletada em Sapé, onde foi constatado um surto, era mais tóxica que a coletada em Jacaraú, onde o produtor informou que anteriormente haviam ocorrido mortes devidas à ingestão dessa planta.

A distribuição geográfica das diferentes espécies tóxicas é um dado importante para o diagnóstico das intoxicações por plantas. A constatação de que na zona da mata Paraibana ocorrem duas espécies que causam sinais semelhantes é importante para que no caso de mortes súbitas seja realizado o diagnóstico diferencial destas duas intoxicações. Neste aspecto, é importante o fato observado na fazenda do município de Jacaraú onde foram encontradas as duas plantas, em áreas diferentes da fazenda.

Agradecimentos.- Este trabalho foi financiado pelo Programa de Apoio a Núcleos de Excelência (Pronex, Proc.001/04), CNPq, FAPESQ, MCT, e pelo Programa Institutos do Milênio (Proc. 420012/2005-2), CNPq. O primeiro autor agradece ao CNPq pela concessão da bolsa de mestrado.

\section{REFERÊNCIAS}

Medeiros R.M.T., Geraldo Neto S.A., Barbosa R.C., Lima E.F. \& RietCorrea F. 2002. Sudden death caused by Mascagnia rigida in cattle in Paraíba, Northeastern Brazil. Vet. Human Toxicol. 44(5):286-288.

Paraguassu A.A. 1983. Intoxicação experimental por Mascagnia rigida Griseb. (Malpighiaceae) em caprinos no Nordeste do Brasil. Dissertação de Mestrado, Univ. Fed. Rural do Rio de Janeiro, Itaguaí, RJ. 65p.

Passos D.A. 1983. Intoxicação experimental em caprinos (Capra hircus) por Palicourea aeneofusca (M.Arg.) Standl. (Rubiaceae). Dissertação de Mestrado, Univ. Fed. Rural de Pernambuco, Recife, PE. 40p.

Riet-Correa F., Medeiros R.M.T. \& Dantas A.F.M. 2006. Plantas Tóxicas da Paraíba. Centro de Saúde e de Tecnologia Rural/ SEBRAE/ PB, Patos, p.1-58.

Tokarnia C.H., Canella C.F.C. \& Döbereiner J. 1961. Intoxicação por um "tinguí" (Mascania rigida Griseb.) em bovinos no Nordeste do Brasil. Arqs Inst. Biol. Animal, Rio de J., 4:203-215.

Tokarnia C.H., Döbereiner J., Couceiro J.E.M. \& Cordeiro Silva A.C. 1983. Intoxicação por Palicourea aeneofusca (Rubiaceae), a causa de mortes súbitas em bovinos na Zona da Mata Pernambucana. Pesq. Vet. Bras. 3(3):75-79.

Tokarnia C.H., Döbereiner J. \& Peixoto P.V. 2000. Plantas Tóxicas do Brasil. Editora Helianthus, Rio de Janeiro, RJ, p.26-27. 